\title{
Energy efficient clustering using the AMHC (adoptive multi-hop clustering) technique
}

\author{
Vimala $M^{1}$, Rajeev Ranjan ${ }^{2}$ \\ ${ }^{1}$ Department of Agricultural Statistics, Applied Mathematics and Computer Science, \\ University of Agricultural Sciences, GKVK Bangalore, India, \\ ${ }^{2}$ REVA University, Bangalore, India
}

\begin{tabular}{l} 
Article Info \\
\hline Article history: \\
Received Oct 26, 2018 \\
Revised Oct 17, 2019 \\
Accepted Oct 28, 2019 \\
\hline
\end{tabular}

\section{Keywords:}

Clustering

IoT

WSN

\begin{abstract}
IoT has gained fine attention in several field such as in industry applications, agriculture, monitoring, surveillance, similarly parallel growth has been observed in field of WSN. WSN is one of the primary component of IoT when it comes to sensing the data in various environment. Clustering is one of the basic approach in order to obtain the measurable performance in WSNs, Several algorithms of clustering aims to obtain the efficient data collection, data gathering and the routing. In this paper, a novel AMHC (Adaptive MultiHop Clustering) algorithm is proposed for the homogenous model, the main aim of algorithm is to obtain the higher efficiency and make it energy efficient. Our algorithm mainly contains the three stages: namely assembling, coupling and discarding. First stage involves the assembling of independent sets (maximum), second stage involves the coupling of independent sets and at last stage the superfluous nodes are discarded. Discarding superfluous nodes helps in achieving higher efficiency. Since our algorithm is a coloring algorithm, different color are used at the different stages for coloring the nodes. Afterwards our algorithm (AMHC) is compared with the existing system which is a combination of Second order data $\mathrm{CC}($ Coupled Clustering) and Compressive-Projection PCA(Principal Component Analysis), and results shows that our algorithm excels in terms of several parameters such as energy efficiency, network lifetime, number of rounds performed.
\end{abstract}

Copyright $(0) 2020$ Institute of Advanced Engineering and Science. All rights reserved.

\section{Corresponding Author:}

Vimala M.,

Department of Electrical and Computer Engineering,

National Chung Cheng University,

168 University Road, Minhsiung Township, Chiayi County 62102, Taiwan, ROC.

Email: vimala.0285@gmail.com, vimalam514@gmail.com, rajeevranjan@reva.edu.in

\section{INTRODUCTION}

IoT is nothing but the network of physical devices embedded with the software sensors, actuators, electronics and connectivity that enable the things in order to connect as well as to exchange the data. It has been observed that the uses of IoT devices has been increased about $31 \%$ every year to approximately 8.4 billion in the year 2017 and it is going to be 30 billion approximately in the year 2020 [1]. IoT has been applicable in the several areas such as smart energy, smart city smart home, smart agriculture etc. Thus, the main aim of IoT is to integrate the physical world to the virtual world. Agriculture is said to be one of the basis for livelihood, the growth of agriculture is considered as backbone of the country's development economically [2,3]. The monitoring system of the agriculture provides the environmental as well as controlling services for the field that leads to the growth of cop [4]. Technology utilization allows us to measure the several factors such as the soil moisture, water level, plant growth condition, humidity. It also tries to improvise the crop productivity [5]. 
In recent years, WSN has been used for the food production and smart agriculture which focuses on the smart agriculture which leads to the rich food production [6]. It also primarily focuses on the monitoring of environmental factor, traceability and equipment [7]. WSN is capable in self- diagnosing, self-configuration, self-organizing and also self-healing, these features of WSN makes a very good choice for the smart agriculture [8]. So, in a simple sentence, WSN is one that refers to the dedicated sensors in order to monitor as well as record the physical conditions of environment and collected data are organized at a central location. Environmental condition such as humidity, pollution levels, temperature, and sound are measured by WSNs [9]. It is mainly comprise of power resources, microcontrollers, sensors, receiver and RF (Radio Frequency) transceivers. However, for smart agriculture, the use of WSN to the IoT is the major driver, and the reason behind is that IoT combines various technology which exists already such as middleware system, WSN, RF identification, cloud computing and the end-user application [10-12].

The IoT helps in agriculture by empowering the farmers with automation technologies and the decision tools that integrates the services, knowledge and products to achieve the profit, better quality and the better productivity $[13,14]$. The below diagram Figure 1 shows the IoT system for agriculture, it has basically five blocks, in which the first block represents the data collection, here the various data such as temperature, humidity, moisture and the other data are collected [15]. The second block represents the transferring of the data, here the data collected data are transferred. Third block presents the device management, basically it is cloud based, so all the data are deployed to the cloud. Henceforth through the application server the data are visualized and monitored. However, the several limitations such as the energy conservation, the limitation of sensors has been always a major factor in extending the life of WSNs, addition to this problem, sensor node also have the few constraints on storage space, communication range, communication bandwidth [16, 17]. Hence the message might be transferred several times through the intermediate notes, this result in energy consumption, message redundancy and traffic collision.

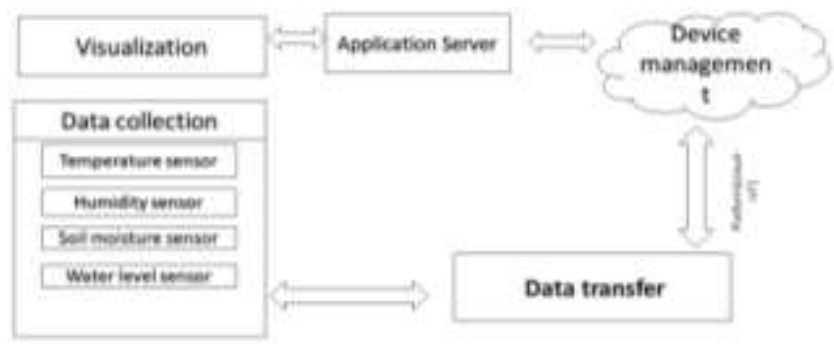

Figure 1. IoT architecture for agriculture

For such problems, the scheme is developed known as AMHC clustering which is being widely used. Through this the WSN can be parted into the various disjointed clusters to take the responsibility of gathering the data and communication process. Hence, in WSN the nodes are required only to gather the information and transmit it to the cluster head, thus huge amount of energy is saved. The main part of this clustering model is to efficiently parting the WSN to disjointed clusters. To optimize the energy conservation, the algorithm of clustering has been proposed.

In order to estimate the performance of WSN, size of cluster is an essential metric [18]. If the size of cluster is small, then the more number of cluster will be available in the WSN, this affects the performance, if the size of cluster is large, then managing the cluster is difficult task for cluster head [19-21]. Through this research, the algorithm is proposed to solve the problem of the above issue. In this paper, a homogenous network is considered so that the graph can be formed as unit disk graph. Henceforth, we propose an algorithm of three phases named as AMHC strategy in order to solve the issue. In first stage, maximal independent set is assembled. In the second stage, extra nodes are coupled. Third stage is for checking and discarding the superfluous nodes. Hence, so it is more suitable for the WSNs, and this particular algorithm is applicable for both general purpose as well as UDG model [22].

The main aim of the existing model was to ensure the energy efficiency and the quality of the data by considering the combination of both SODCC and CCPCA, but it fails marginally in terms of performance. The disadvantage of the existing model is, it fails to deliver the satisfactory result, and for example the delay time is so, high that it is difficult to consider when comes to the real time scenario. Other primal disadvantage is that it consumes lot of energy for the quality of data, this makes system expensive. This particular research is organized such as: In section two, the literature is presented. Section 3 is all about proposed models, simulation and results are written in fourth section. The conclusion as well as future work are described in the last section of this paper 


\section{LITERATURE SURVEY}

In this section of the research, several previous work is mentioned which helped in developing the proposed system. O. Younis and S. Fahmy [23], in this research, an energy efficient, distributed approach was presented for the ad hoc networks, here the protocol named as HEED is presented, which selects the cluster heads in accordance with the hybrid of NRE (Node Residual Energy) and parameters such as node proximity to their node degree is also selected. The HEED protocol that terminates in a unchanged number of iterations. The parameters of HEED such as network operational interval and minimum selection probability can be used for optimizing the resource usage in accordance to the application requirement and network density. HEED protocols tries to achieve the connected multihop inter-cluster network but it was applicable only for the small network. M. Demirbas, et al [24], a FLOC is presented, which parts the multi-hop wireless network to equal sized and the overlapping clusters. Here, each cluster contains the cluster head, and they are situated such that the nodes within the unit distance of cluster head exists in the cluster head and none of the node beyond the particular distance $\mathrm{m}$ from cluster heads belongs to the particular cluster. The locality is achieved by asserting $m>=2$ in FLOC. Regardless in the network size, the FLOC successfully exploits the particular nature of the wireless-radio model and hence obtains the clustering, although it tries to achieve regardless of the network size, but the outcome were not satisfactory.

J. Qiao and X. Zhang [25], the method of compressed data gathering is proposed in order to get rid of the problem of unbalanced position and the random selection. Even Clustering method is proposed based on the location, clustering is applied with the similar size of grids that ensures the positional balance. In case of uneven nodes, density based clustering is proposed. In DEC method, the factors such as density and location are considered and it equalizes the energy, extends the network lifetime and reduces the energy consumption. Several factors such as the environmental factor and the node sizes are ignored though. S. Hu and G. Li, [26], to avoid the failure of WSNs, RH (regular-hexagonal) clustering scheme of the sensor networks and hence analyze the model. Henceforth, the SFT (Scale Free topology) evolution mechanism is presented, later the characteristics of SFTEM using the mean-field theory. This system only saves WSN from its failure, but do not provide the efficient mechanism.

J. Zhou, et al [27], CDS (Connected dominating set) is proposed to serve as backbone of WSN, because there might be failure in sensor node due to various reasons. So, it is essential to design a fault tolerant along with the high redundancy in connectivity as well as coverage. So in this paper the algorithm is proposed named as $\gamma$-approximation for the CDS problem, it also gives the improvement in the performance ratio of given approximation algorithm on the UDG. [28] Almost all the approximation algorithm follows a two phased scheme in order to construct the CDS in network. In first phase, the DS (dominating SET) is constructed and in the second phase the nodes available are connected. MIS (Maximum Independent Set) is used as DS, thus the relation among the MCDS and MIS plays an essential role. In case of Homogenous network, the ad hoc networks are modeled as UDG (Unit Disk Graph) as well as UBG (Unit Ball Graphs) and in case of heterogeneous network; it is modeled as DGB and BGB. So, in this paper we focus on the problems of UB (Upper Bound) for the size of MISs in the heterogeneous network (wireless). To achieve this classical mathematics problem such as sphere packing and circle packing problem is used. R. Misra and C. Mandal [29], the MCDS problem is the NP-complete in the UDG, hence many heuristic based DAA (Distributed Approximation Algorithm) are used. To enhance the performance ratio a new method was introduced, which was based on two principles. First principle is that the domatic number of the connected graph should be two, second principle is that the OSS (Optimal substructure set) of independent set prefers with the CC (Common Connector).Thus, PST (Partial Steiner Tree) is achieved while constructing the independent sets. Afterwards the final post processing steps recognizes the Steiner nodes during the formation of Steiner tree for the IS (Independent sets). The data collected should be efficiently aggregated by the sensor is very much essential for the WSNs. In this research, the Design time-EA (efficient aggregation) algorithm is thoroughly studied. An efficient algorithm is proposed which produces DAT (data aggregation tree) and CFA (collision free Aggregation) schedule, the latency of the aggregation is bounded by the time slots also the LB (lower bound) is derived for the aggregation.

Several clustering algorithm has been presented in order to provide the higher efficiency, however all these algorithm and the scheme lacks the efficiency. In the paper described either the several factors regarding the clustering, factors such as location, density is ignored or they do not provide the desired outcome. On average almost all the paper discussed have ignore $d$ the node size so, in order to overcome these problems we have proposed the methodology which is discussed in the next section of this research. 


\section{PROPOSED METHODOLOGIES}

\subsection{System model}

Here, it is assumed that all the nodes in the WSNs are distributed in a 2D-plane and it has an equal maximal transmission range of single unit. Graph is represented by $U=(X, Y)$. X represents the sensor node set and $\mathrm{Y}$ represents the edges. An edge $(\mathrm{a}, \mathrm{b}) \in \mathrm{Y}$ and $\mathrm{a}, \mathrm{b}$ are the transmission range of each other's. Our proposed algorithm consists of three stages, which helps to discard superfluous nodes. The below diagram i.e. Figure 2 shows the proposed architecture of our model, it consists of base station, cluster heads, nodes. The nodes are connected to their respective cluster head, and this cluster heads are connected to the base station, they are connected with the multi hop routing.

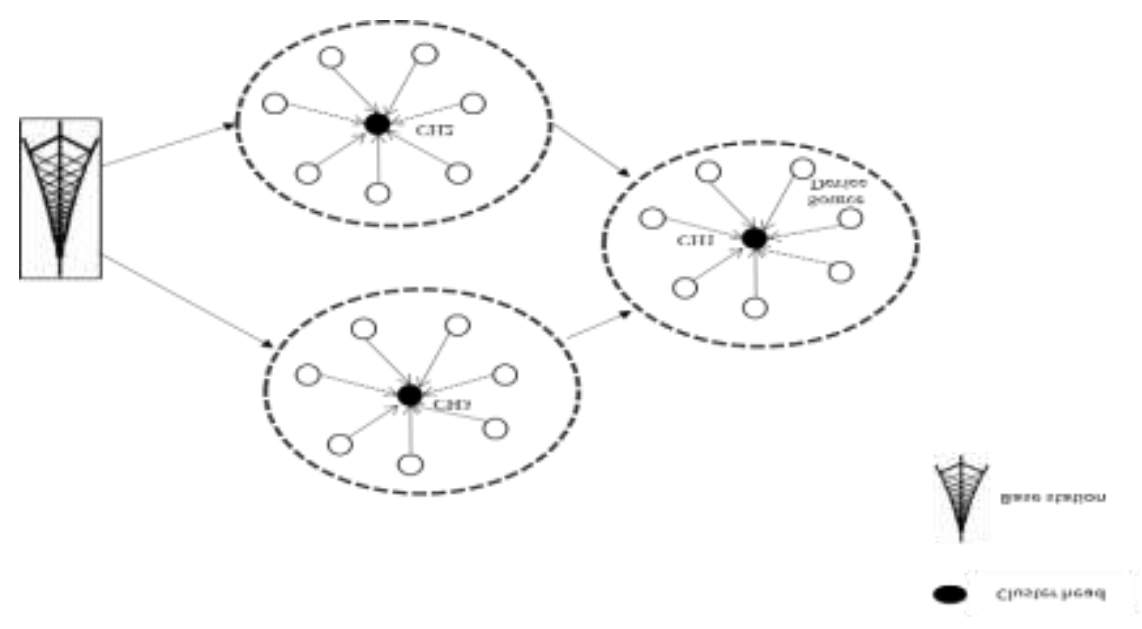

Figure 2. Proposed architecture model

\subsection{AMHC (Adoptive multi-hop clustering) algorithm}

The proposed algorithm is named as the AMHC (Adaptive Multi Hop Clustering) algorithm in $\mathrm{U}=(\mathrm{X}, \mathrm{Y})$. AMHC algorithm is the coloring algorithm, in this case we usually use the four different color for denoting. The white, blue, grey, black color nodes are denoted as $W_{c}, B_{C}, G_{c}, B K_{c}$ respectively. At first all the nodes are $W_{c}$, when the nodes are selected as the dominator, color is $B K_{c}$, and when the neighbor nodes are dominated by the $B K_{c}$, then it is $G_{c}$. $B_{C}$ Nodes are the one which is used for connecting the dominators. AMHC algorithm contains has three stage, they are assembling, coupling, removing.

\subsubsection{First stage: Assembling of maximum-IS (Independent sets)}

The maximum independent also known as dominating set (ds) in the given graph. The node in maximum independent set is selected one by one. The first stage known as the assembling stage shows the steps involved while constructing the maximum independent sets. In first stage, the connected graph $\mathrm{U}=(\mathrm{X}, \mathrm{Y})$ is taken as the input and the expected output is the maximum independent sets of connected graph $\mathrm{U}$.

\section{Assembling algorithm}

Step1: node in $X$ are $W_{c}$

Step2: let's consider that B is Null

Step3: while (there exist a $W_{c}$ in $X$ )

Do

Step4: find $W_{c}$ node a from $X \backslash B$ such that within the d hops, the number of a's $W_{c}$ neighbors are maximum.

To break the ties use id;

Step5: in this step, the node a is $B_{C}$ node and the remaining $W_{c}$ node after this step is $G_{C}$

Step6: $B=B U\{a\}$;

Step7: return $B$.

The algorithm is said to be the greedy algorithm, at first the all node in $\mathrm{X}$ are $W_{c} \mathrm{r}$. Later, for the $W_{c}$ node a, the number of a's $W_{c}$ nodes within the d hops are calculated. From all these $W_{c}$ nodes, the node with the maximum degree is selected. The main intention here is to divide and conquer, in this extra nodes are added 
in order to make B more nearer. The idea is to divide and conquer, the assembling algorithm consists of several procedure, each procedure has sub-procedure, and again these sub-procedure have the sub-procedure. The subset $D_{i}$ is chosen from the $X \backslash E_{j<i} D_{i} U B$ such that $E_{j \leq i} D_{i} U B$ is the dominating sets. The definition of $\mathrm{P}$ (i) is shown in (1).

$$
\begin{aligned}
& P(0)=2 d+1 \\
& P(i)=\left\lfloor\frac{P(i-1)+1}{2}\right\rfloor
\end{aligned}
$$

Since $\mathrm{P}$ (i) is MDF (Monotone Decreasing Function), the nodes in the $\mathrm{P}(\mathrm{i})$-hop is more nearer than in $\mathrm{P}(\mathrm{i}-1)$. After the assembling algorithm, B is assumed as the $\mathrm{P}(0)$ hop-ds(dominating sets), that means the two nodes in $\mathrm{B}$ are connected by $(2 \mathrm{~d}+1)$. After the termination of the above algorithm, the single-hop connected ds (dominating set) are generated.

\subsubsection{Second stage: Coupling the maximum-IS (Independent Sets)}

After assembling, the Maximal-IS (independent sets) is obtained, and it is denoted as B. In the second stage the input taken is the connected graph $\mathrm{U}=(\mathrm{X}, \mathrm{Y})$ and the output of first stage. The expected output after the second stage is connected dominating sets.

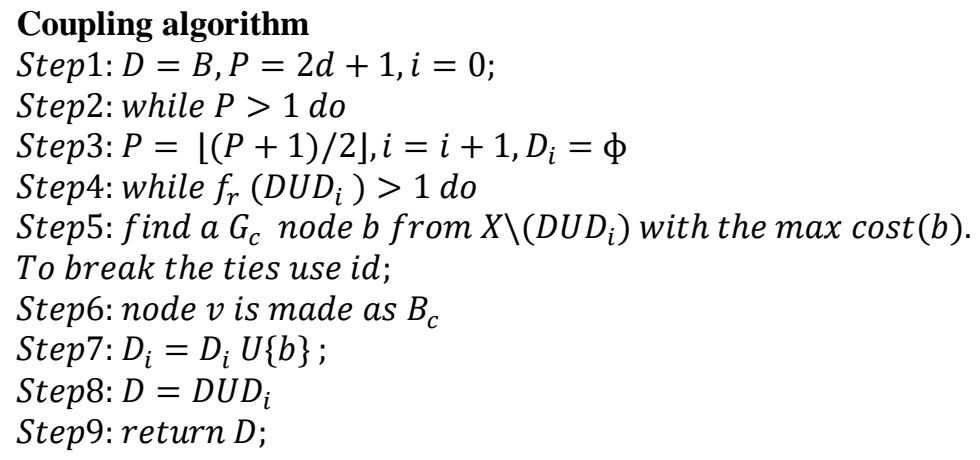

In the algorithm the main intention is how the nodes in $D_{i}$ is determined. In any given graph, $\mathrm{U}=(\mathrm{X}, \mathrm{Y})$, two nodes $\mathrm{a}, \mathrm{b} \in \mathrm{X}$ are said to be the h-hop connected only if there is existence of any path in the particular graph $\mathrm{U}$ and length is h. In order to make the $C_{i}$ size small, the most efficient nodes are selected which makes $E_{j \leq i} D_{i} U B$ to form $\mathrm{P}$ (i)-connected ds (dominating sets). The main moto here is to select the nodes iteratively that minimizes the P (i)-hop CC (Connected Components). Moreover, to make this particular algorithm economical, the fewest nodes are considered. The second stage is described below. At first, the $i^{t h}$ iteration of the $i^{\text {th }}$ round, the $D_{i}$ has $\mathrm{t}-1$ nodes. And let (2) defines the D.

$$
D=E_{j \leq i} D_{i} \leq B
$$

In $X \backslash D$, for a node $\mathrm{v}$ the $\mathrm{P}$ (i)-hop $\mathrm{CC}$ (Connected components) are reduced by $-\Delta_{v} f_{r(i)}(D)$. Hence no. (b) is used to denotes the whole number of nodes in the shortest paths. $\cos t(b)$ is used for denoting the cost of $b$.

$$
\operatorname{cost}(b)=\frac{-\Delta_{b} f_{r(i)}\left(D U D_{i}\right)}{n o .(b)}
$$

Here, the node, which has the largest cost, is selected.

\subsubsection{Stage 3: Discarding the superfluous nodes:}

After the stage 1 and stage 2, the common ds(dominating sets) are achieved and it is denoted as the $\mathrm{C}$. Nodes in $\mathrm{C}$ are either $B K_{c}$ or $B_{c}$. Hence it is easy to find the existing superfluous nodes in $\mathrm{C}$. In stage 3 the input taken are connected graph and the outcome of second stage, the possible outcomes are the smaller connected-DS (Dominating sets) of the graph. In this particular algorithm the main idea is to further minimize the size of D through checking and discarding the superfluous nodes that exist in D. Hence, according to 
the minimum connected-ds(dominating sets) the node a in connecting-ds(dominating sets) is superfluous if it satisfies the following two criteria.

1. Each and every node which is dominated by node should have one AD (Alternative Dominator).

2. Sub Graph that are prompted by the $\mathrm{D}-\{\mathrm{a}\}$ is coupled.

The first need guarantees the dp (domination property), i.e. once the superfluous nodes are discarded, then the nodes that are available still dominates the complete network within the hops, here the dominator refers either $B_{c}$ or $B k_{c}$. The second need is analyzed such that, in any node $a \in D$, there arises two scenario, first scenario is that the sub Graph U[D] is prompted by D, if a is the leaf node, then discarding a does not have any effect on the sub graph connectivity. Second scenario is, if a is coupled with the more than one connectors, then the given sub graph is coupled only when the other connectors are also coupled. In order to check whether these given connectors are connected or not, the complete sub graph $U[D-\{a\}]$ might be involved, so the TC (Time Complexity) is large.

\author{
Discarding algorithm \\ Step $1: L \leftarrow\left\{b \mid b\right.$ is the $B K_{C}$ node in $\left.U[D]\right\}$; \\ Step2: while there exists a superfluous node $b \in N$ do \\ Step3: make node $b$ as $G_{c}$, remove b from $D$; \\ Step4: If $X^{\prime}$ s direct connector a is leaf in $U[D]$ then \\ Step5: make a as $B K_{c}$ and add a into $N$. \\ Step6: return D.
}

Moreover, the size of $\mathrm{D}$ is reduced by the discarding algorithm, here in each iteration the $B K_{C}$ nodes are considered, these nodes are basically leaves in the sub graph of $U$ [D].

\title{
4. SIMULATION RESULT AND ANNALYSIS
}

The system configuration used in this research is windows 10 enterprises operating system along with 64 bit quad core processor, 2GB NVDIA graphics packed with 16 GB of RAM. Dot net based simulator known as sensoria simulator is used which uses the $\mathrm{C}$ sharp programming language. The Simulation is conducted based on the several parameters for energy efficiency, network lifetime and henceforth we compared this parameter with existing LEACH based algorithm. Moreover, Table 1 presents the various network parameter for simulation, here we have considered the praemeter such as Network size, number of sensor nodes used, and here sensor nodes used is 200, 400, 600 and 800. other parameter such as Number of Base Station, Initial energy of the nodes, length of packet, TS(Transmission Speed ), bandwidth, processing delay and few other parameters are mentioned which helps in getting the ideal simulation environment.

Table 1. Network parameter

\begin{tabular}{ll}
\hline Network parameter & Value \\
\hline Size of the network & $25 \mathrm{~m} * 25 \mathrm{~m}$ \\
sensor nodes used & $200,400,600,800$ \\
Base Station used & 1 \\
Energy(Initial) of the sensor nodes & $0.2 \mathrm{~J}$ \\
Radio-ed (energy dessipiation) & $50 \mathrm{nj} / \mathrm{bit}$ \\
Data -PL(Packets Length) & $2000 \mathrm{bits}$ \\
Transmission speed & $100 \mathrm{bit} / \mathrm{s}$ \\
Bandwidth & $5000 \mathrm{bit} / \mathrm{s}$ \\
Consumption of Idle energy (Eelec) & $50 \mathrm{nj} / \mathrm{bit}$ \\
Data packet-PD(Processing Delay) & $0.1 \mathrm{~ms}$ \\
Amplification energy (Emp) & $100 \mathrm{pJ} / \mathrm{bit} / \mathrm{m} 2$ \\
\hline
\end{tabular}

The Tables 2-4 presents the value comparison with the existing system which is combination of second -order DCC (Data coupled Clustering) and Compressive- Projection PCA( Principal component analysis) also known as SODCC and CPPCA respectively. In the Tables 2-4, the improvement in the proposed method is calculated in percentage. In Table 2 we see that as the number of nodes increases, the improvisation in the performance over the existing takes place. Table 3 shows the number of failed nodes, as the number of nodes increases, the failed nodes increases automatically in case of existing while it keeps decreasing in case of proposed and there is marginal improvement. Table 4 gives the idea about the number of rounds performed and it is calculated in percentage, for 200 nodes, it is $71.23 \%$ and as the number of nodes increases the improvement in percentage goes high and it reaches up to $95.46 \%$. 
Table 2. End-to-end time delay

\begin{tabular}{cccc}
\hline & & End to end time delay & \\
Nodes & Existing & 101.8126 & Improvement in terms of \%(percentage) \\
\hline 200 & 144.4769 & 94.00895 & 22 \\
400 & 120.4473 & 91.44554 & 33 \\
600 & 136.4326 & 91.35604 & 48 \\
800 & 174.4836 & & 40 (AMHC) \\
\hline
\end{tabular}

Table 3. Number of failed nodes

\begin{tabular}{|c|c|c|c|}
\hline Nodes & Existing & $\begin{array}{l}\text { Number of failed nodes } \\
\text { Proposed (AMHC) }\end{array}$ & Improvement in terms of $\%$ (percentage) \\
\hline 200 & 31 & 9 & 70.96 \\
\hline 400 & 16 & 9 & 43.75 \\
\hline 600 & 29 & 8 & 72.41 \\
\hline 800 & 49 & 8 & 83.67 \\
\hline
\end{tabular}

Table 4. Comparison after the first sensor node death

\begin{tabular}{cccc}
\hline & \multicolumn{2}{c}{ First sensor node death (number of rounds performed) } \\
Nodes & Existing & Proposed(AMHC) & Improvement in terms of \%(percentage) \\
\hline 200 & 233 & 810 & 71.23 \\
400 & 118 & 855 & 86.19 \\
600 & 105 & 1159 & 90.94 \\
800 & 54 & 1191 & 95.46 \\
\hline
\end{tabular}

The Figure 3 and Figure 4 shows the number of failed nodes and the end to end time delay i.e. processing delay respectively when there is a death of $30 \%$ of sensor node. The graph of the same shows that our proposed AMHC algorithm preforms better than the existing LEACH algorithm at different sensor nodes. From the Figure 3 and Figure 4, it is clear that when end to end delay keep getting reduced as the number of nodes increases which proves the efficiency of our algorithm when compared to the leach algorithm.

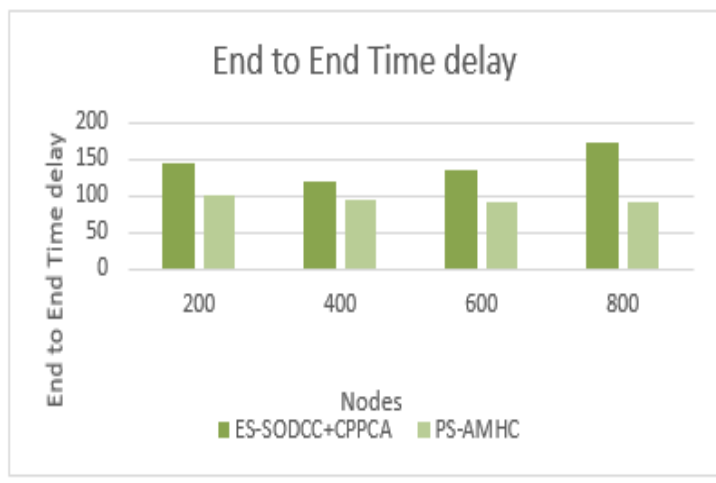

Figure 3. End to end time delay comparison

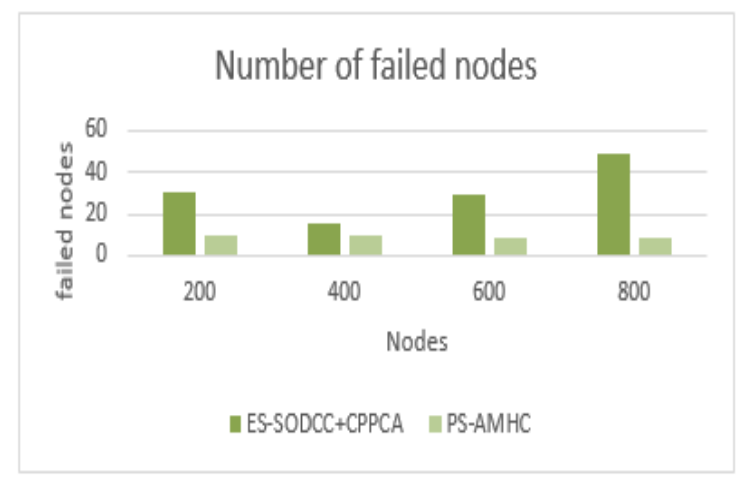

Figure 4. Number of failed nodes

Similarly, Figures 5-8 shows the number of rounds performed by the existing leach algorithm and AMHC algorithm respectively, after the death of the first sensor node for the various number of nodes. In Figure 5 it is shown that the proposed algorithm outperforms the leach algorithm, the number of rounds performed by leach scheme is for 200 sensor node is 233, whereas AMHC algorithm performs 810 rounds. In case of first sensor node death for 400 nodes, the number of rounds is 118 and 855 for LEACH and AMHC scheme respectively as shown in Figure 6. In case of first sensor node death for 600, the number of rounds performed is 105 and 1159 for existing algorithm and proposed algorithm as shown in Figure 7. Similarly, the comparison between these two algorithms are done for 800 nodes in and number of rounds is marginally big i.e. 54 and 1159 respectively in Figure 8. 


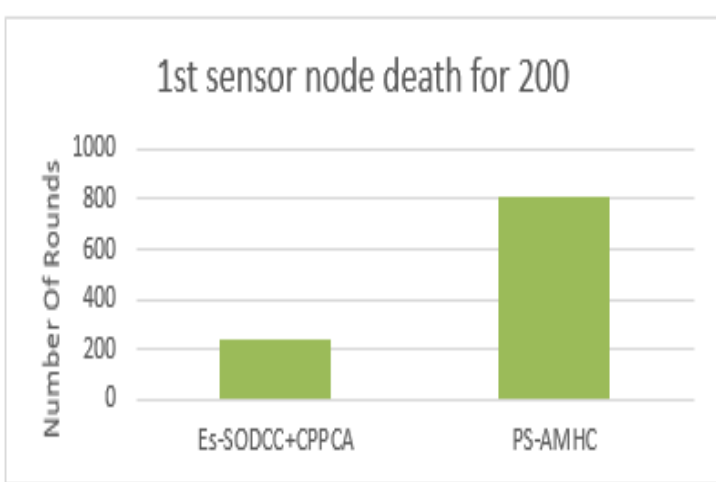

Figure 5. Number of rounds after $1^{\text {st }}$ sensor node death

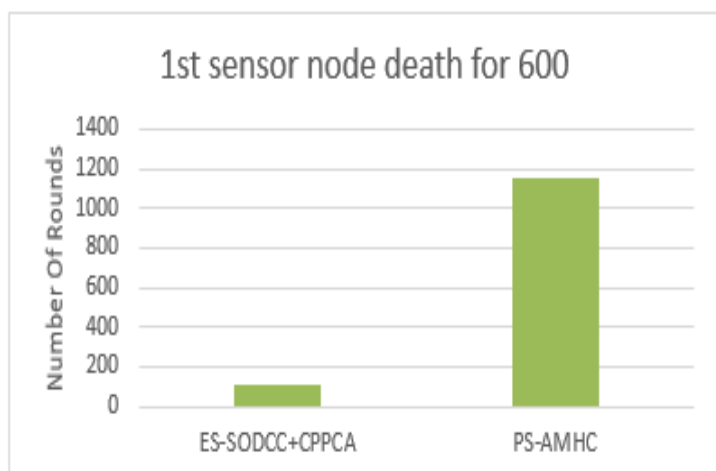

Figure 7. Number of rounds after $1^{\text {st }}$ sensor node death

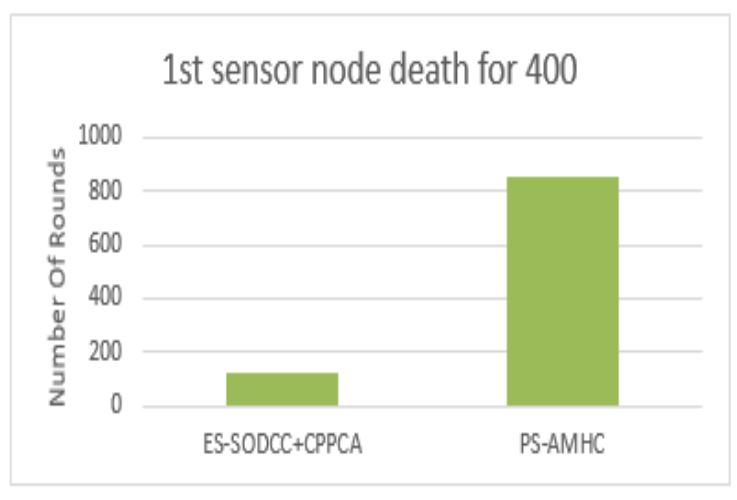

Figure 6. Number of rounds after $1^{\text {st }}$ sensor node death

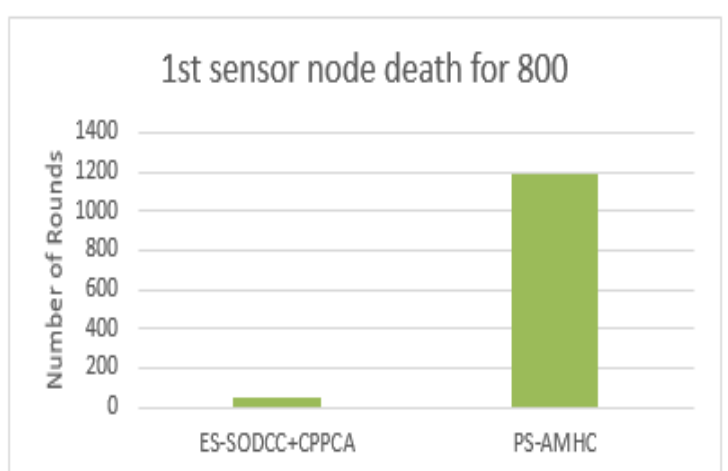

Figure 8. Number of rounds after $1^{\text {st }}$ sensor node death

Figures 9-12 shows the comparison of existing system with the proposed system in terms of lifetime of network after death of $75 \%$ node. Figure 9 shows the network lifetime of a network after the death of $75 \%$ nodes for the 200 sensor nodes and it is little margin, Figure 10 shows the network lifetime for 400 nodes and similarly Figure 11 and Figure 12 shows the lifetime of a network for 600 and 800 respectively. With these three graphs, it has been observed that as the node increases the performance of the AMHC algorithm also marginally increases when compared with the leach algorithm.

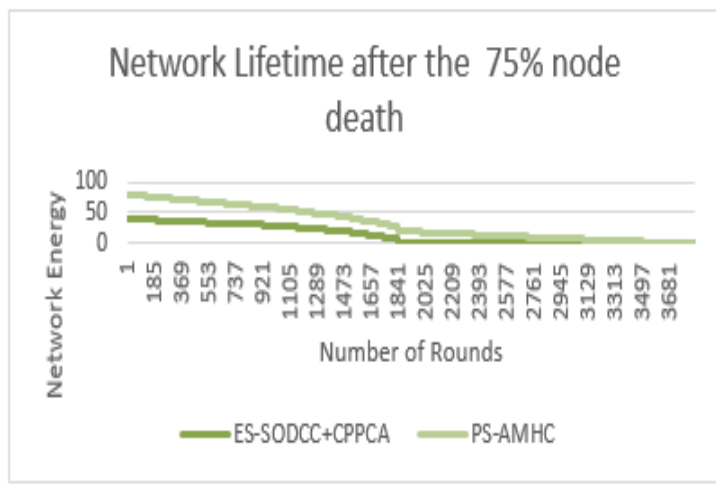

Figure 9. Network lifetime after the death of $75 \%$ node

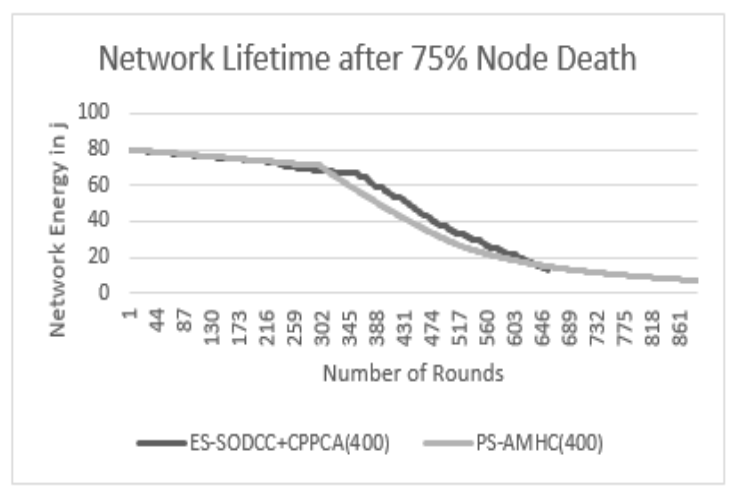

Figure 10. Network lifetime after the death of $75 \%$ node 


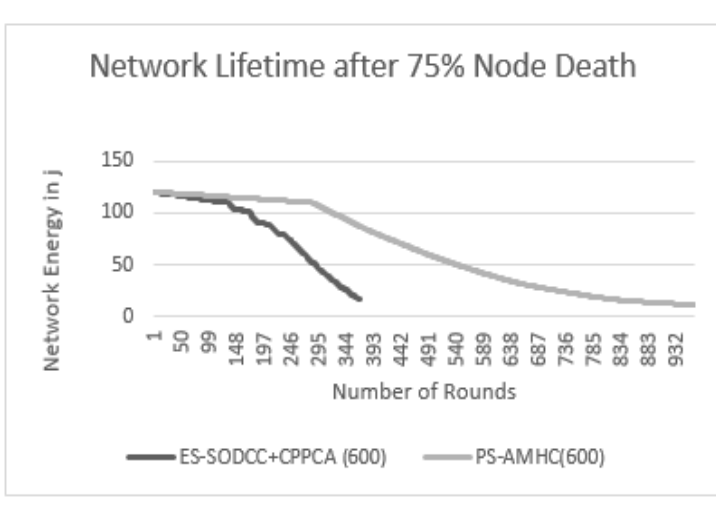

Figure 11. Network lifetime after the death of $75 \%$ node
Network Lifetime after 75\% Node Death

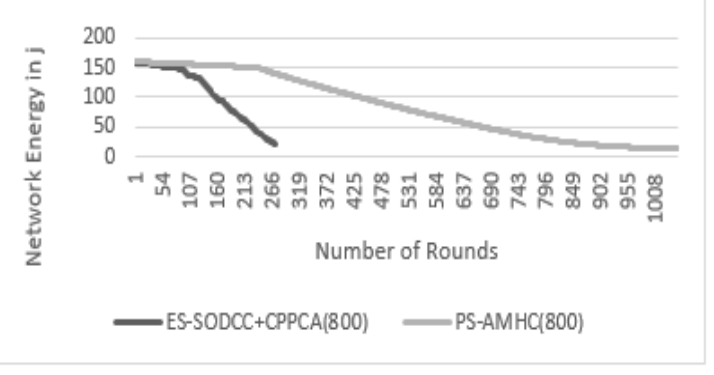

Figure 12. Network lifetime after the death of $75 \%$ node

\section{CONCLUSION}

Clustering is the technique, which was proposed to provide the efficient platform for the network topology in order to extend the lifetime of a network. Since most of the existing algorithm overlook the performance of network and the problem of multi-hop connection are ignored. So, in this paper, in any given homogenous network the problem of the network is analyzed and later an Adaptive Multi Hoping Clustering (AMHC) is proposed. The algorithm of AMHC consists of three stages namely assembling, coupling and discarding the superfluous nodes. In first stage, the distance between the neighboring nodes are made large and the maximal-IS (Independent Sets) are assembled, the second stage involves in coupling the maximal-IS (Independent Sets). Third and final stage involves in discarding the superfluous nodes. Later, the Our proposed algorithm AMHC is compared with the existing algorithm(SODCC+CPPCA) in terms of various parameter such as number of failed nodes, end to end time delay, number of rounds performed at the different nodes when first sensor node is dead and the lifetime of network is also compared with the leach algorithm. The comparison clearly shows that our algorithms outperforms the existing algorithms and it excels. When observed in terms of percentage the number of rounds performed reaches up to $95.46 \%$. To achieve the high efficiency, the data gathering, data collection and routing is very much essential aspects, so to achieve that in future several scenario can be focused.

\section{REFERENCES}

[1] N. Wang, N. Zhang, and M. Wang, "Wireless sensors in agriculture and food industry-recent development and future perspective," Computers and electronics in agriculture, vol. 50, no. 1, pp. 1-14, 2006.

[2] Pushpa, S.K., Ramachandran, S., \& Kashwan, K.R. (2014). A Novel Skeleton Extraction Algorithm for 3d Wireless Sensor Networks..

[3] Desai, S.C., Jagadeesh, K.S., \& Dhruve, K.D. (2013). ELEPHANT SWARM OPTIMIZATION FOR WIRELESS SENSOR NETWORKS -A CROSS LAYER MECHANISM.

[4] y Chandramouli.H, Dr. Somashekhar C Desai, K S Jagadeesh \& Kashyap D Dhruve, "Enhancing Network Lifetime in Wireless Sensor Networks Adopting Elephant Swarm Optimization," Global Journal of Computer Science and Technology Network, Web \& Security, vol. 13, no. 4 Version 1.0 Year 2013 Type: Double Blind Peer Reviewed International Research Journal Publisher: Global Journals Inc. (USA) Online ISSN: 0975-4172 \& Print ISSN: 0975-4350.

[5] O. Elijah, I. Orikumhi, T. A. Rahman, S. A. Babale, and S. I. Orakwue, "Enabling Smart Agriculture in Nigeria: Application of Iot and Data Analytics," in 2017 IEEE 3rd International Conference on Electro-Technology for National Development (NIGERCON), pp.762-766, Nov 2017.

[6] N. Wang, N. Zhang, and M. Wang, "Wireless Sensors In Agriculture and Food Industry-Recent Development and Future Perspective," Computers and electronics in agriculture, vol. 50, no. 1, pp. 1-14, 2006.

[7] G. Mahalakshmi and V. M. Bhaskaran, "Managing mobility in wireless cellular networks: A profile based approach," 2014 International Conference on Electronics and Communication Systems (ICECS), Coimbatore, 2014, pp. 1-5..

[8] S. E. Díaz, J. C. Pérez, A. C. Mateos, M.-C. Marinescu, and B. B. Guerra, "A Novel Methodology for the Monitoring of the Agricultural Production Process based on Wireless Sensor Networks," Computers and Electronics in Agriculture, vol. 76, no. 2, pp. 252-265, 2011.

[9] Vijayalakshmi, Meeniga and Linganagouda Kulkami. "Quality aware protocol to support multimedia data delivery in wireless network," 2016 3rd International Conference on Devices, Circuits and Systems (ICDCS) (2016), pp. 322-326. 
[10] F. TongKe, "Smart Agriculture based on Cloud Computing and Iot," Journal of Convergence Information Technology, vol. 8, no. 2, 2013.

[11] https://academic.oup.com/jxb/article-abstract/63/1/13/553113?redirectedFrom=fulltext.

[12] S. S. Sodhi, A. S. Kunwar, K. Dhingra and G. Suganya, "Smart chair," 2017 International Conference on Inventive Computing and Informatics (ICICI), Coimbatore, pp. 139-148, 2017.

[13] A. Behura, A. Narayan, A. K. Ray and S. K. Pani, "A complete model for IOT application," 2017 International Conference on Intelligent Sustainable Systems (ICISS), Palladam, pp. 1026-1030, 2017.

[14] https://www.ncbi.nlm.nih.gov/pmc/articles/PMC5134543/

[15] Y. E. M. Hamouda and B. H. Y. Elhabil, "Precision Agriculture for Greenhouses Using a Wireless Sensor Network," 2017 Palestinian International Conference on Information and Communication Technology (PICICT), Gaza City, pp. 78-83, 2017.

[16] B. Patil and R. Kadam, "A Novel Approach to Secure Routing Protocols in WSN," 2018 2nd International Conference on Inventive Systems and Control (ICISC), Coimbatore, India, pp. 1094-1097, 2018.

[17] https://www.researchgate.net/publication/305869497_Internet_of_Things_A_review_from_\%27Farm_to_Fork\%27

[18] C. S. Nam, Y. K. Ku, J. W. Yoon and D. R. Shin, "Cluster Head Selection for Equal Cluster Size in Wireless Sensor Networks," 2009 International Conference on New Trends in Information and Service Science, Beijingpp. 618-623, 2009.

[19] Goutam Chakraborty, "A Note on cluster size of WSN," 2009 4th International Conference on Computers and Devices for Communication (CODEC), Kolkata, pp. 1-6, 2009.

[20] V. Pal, G. Singh and R. P. Yadav, "Balanced Cluster Size Solution to Extend Lifetime of Wireless Sensor Networks," in IEEE Internet of Things Journal, vol. 2, no. 5, pp. 399-401, Oct. 2015.

[21] C. Sevgi and A. Kocyigit, "On determining cluster size of randomly deployed heterogeneous WSNs," in IEEE Communications Letters, vol. 12, no. 4, pp. 232-234, April 2008. doi: 10.1109/LCOMM.2008.071942.

[22] Heinz Breu, David G. Kirkpatrick, "Unit disk graph recognition is NP-hard, Computational Geometry," vol. 9, no. 1-2, 1998.

[23] O. Younis and S. Fahmy, "Heed: a hybrid, energy-efficient, distributed clustering approach for ad hoc sensor networks," IEEE Transactions on Mobile Computing, vol. 3, no. 4, pp. 366-379, 2004.

[24] M. Demirbas, A. Arora, and V. Mittal, "Floc: A Fast Local Clustering Service for Wireless Sensor Networks," in DIWANS/DSN, 2004.

[25] J. Qiao and X. Zhang, "Compressive Data Gathering Based on Even Clustering for Wireless Sensor Networks," in IEEE Access, vol. 6, pp. 24391-24410, 2018.

[26] S. Hu and G. Li, "Fault-Tolerant Clustering Topology Evolution Mechanism of Wireless Sensor Networks," in IEEE Access, vol. 6, pp. 28085-28096, 2018.

[27] J. Zhou, Z. Zhang, S. Tang, X. Huang, Y. Mo and D. Z. Du, "Fault-Tolerant Virtual Backbone in Heterogeneous Wireless Sensor Network," in IEEE/ACM Transactions on Networking, vol. 25, no. 6, pp. 3487-3499, Dec. 2017.

[28] S. Bai, X. Che, X. Bai and X. Wei, "Maximal Independent Sets in Heterogeneous Wireless Ad Hoc Networks," in IEEE Transactions on Mobile Computing, vol. 15, no. 8, pp. 2023-2033, Aug 1, 2016.

[29] R. Misra and C. Mandal, "Minimum Connected Dominating Set Using a Collaborative Cover Heuristic for Ad Hoc Sensor Networks," in IEEE Transactions on Parallel and Distributed Systems, vol. 21, no. 3, pp. 292-302, March 2010 